\title{
CORONAVIRUS
}

\section{Pregnancy influences immune responses to SARS-CoV-2}

\author{
Cristian Ovies $^{1,2}$, Eleanor C. Semmes ${ }^{1,2}$, and Carolyn B. Coyne ${ }^{1,2^{*}}$ \\ 1Department of Molecular Genetics and Microbiology, Duke University School of Medicine, Durham, NC, USA \\ 2Duke Human Vaccine Institute, Duke University School of Medicine, Durham, NC, USA \\ *Corresponding author. Email: carolyn.coyne@duke.edu
}

Pregnancy and fetal sex influence the quality of antibody responses to SARS-CoV-2 infection and immunization (Atyeo et al., Bordt et al.).

For over a year, the severe acute respiratory syndrome coronavirus 2 (SARS-CoV-2) pandemic has challenged our clinical and scientific expertise, exposing the shortcomings in our understanding of the virus as well as the gaps in healthcare delivery and access worldwide. During the coronavirus disease 2019 (COVID-19) pandemic, numerous studies have investigated the innate and adaptive immune responses to SARS-CoV-2 infection and vaccination in different clinical cohorts; however, relatively few studies included lactating and pregnant women, a highly vulnerable population for the development of severe COVID-19. Moreover, pregnant women have been sidelined during the clinical trials that eventually led to the approval of the mRNA-1273 and BNT162b2 SARS-CoV-2 vaccines $(1,2)$. This is not an isolated occurrence, as pregnant women have historically been excluded from the majority of clinical trials, limiting their representation in the development of therapeutics and restricting our understanding of how different stages of pregnancy modulate immune responses to infections and vaccinations (3). This is particularly important given that infections during pregnancy are common contributors to maternal mortality and morbidity (4).

Two recent studies in Science Translational Medicine by Atyeo et al. (5) and Bordt et al. (6), investigate how pregnant women respond to SARS-CoV-2 mRNA vaccines and natural infection, respectively. These studies demonstrate that pregnant and lactating women mount robust antibody responses to vaccination and infection. However, factors such as time of vaccine priming and boosting as well as fetal sex modulate maternal antibody responses and passive immunity conferred on the fetus.

\section{Pregnancy as a Unique Immunological State}

Pregnancy represents a unique biological state in which a balance of immunotolerance toward the developing fetus must be achieved while also protecting the pregnant woman against infectious diseases (7). This balancing act is nontrivial to achieve given that the immune system in pregnancy can be influenced by both maternal and fetal components such as the placenta. The placenta is a fetal-derived organ and thus can be of male or female origin. Due to the numerous systemic changes in innate and adaptive immunity that occur throughout gestation, pregnant women are more susceptible to severe disease resulting from pathogens including SARSCoV-2 and influenza virus (7). However, timely maternal immunization against influenza and pertussis can induce a robust immune response that can protect the pregnant woman directly and the fetus through passive antibody transfer. Defining how pregnant and lactating women respond to SARSCoV-2 infection and vaccination is thus critical to optimize vaccination strategies that protect both mother and infant from COVID-19.

One of the primary contributors to fetal and neonatal immunity is passive transfer of maternal antibodies across the placenta and through breastmilk, which equips the infant with an arsenal of functional immunoglobulins (Ig) (8). Maternal IgG antibodies are transferred to the fetal circulation by binding to Fc-domain receptors (FcRs) on the surface of the syncytiotrophoblast, the multinucleated cell layer overlying the placenta. The neonatal Fc receptor $(\mathrm{FcRn})$ is the primary placental IgG transfer receptor, but other placentallyexpressed $\mathrm{Fc} \gamma$ receptors also modulate transfer efficiency. Thus, antibody-FcR binding is a key determinant of transplacental transfer and differential glycation of the antibody Fc-domains can influence the quantity of antibody transferred (8). Following parturition, mothers transfer IgA-isotype antibodies through breastmilk, which can protect infants against enteric and respiratory infections. Once in the neonatal circulation, maternal antibodies can coordinate diverse immune functions. These include direct antigen neutralization and non-neutralizing, cell-mediated functions (Fig. 1A), many of which are orchestrated by Fc $\gamma$ Rs. The diversity of antibody functions demonstrates their unique ability to provide comprehensive immunological protection to fetuses and breastfeeding infants.

Naturally acquired infection and prophylactic immunization are distinct pathways of antigen exposure that generate diverse antibody responses. Therefore, resultant antibodies might differ based on the method of antigen exposure. In pregnant women, these differences can alter the functional nature of antibodies and affect their biological activity and 
capacity to be delivered to the fetus or infant.

Antibody Quality in the Context of Pregnancy and SARS-CoV-2 Immunization

Using a systems serology approach, Atyeo et al. immunophenotyped anti-SARS-CoV-2 antibodies in the sera of nonpregnant, pregnant, and lactating women following immunization with the mRNA-1273 or BNT162b2 COVID-19 vaccines (Fig. 1B). Compared to immunized non-pregnant women, pregnant and lactating women exhibited overall lower SARSCoV-2 antibody titers, restricted IgG subclass responses, and a decreased FcR binding capacity following the first dose ("prime") of vaccination. However, following the second dose ("boost"), there were minimal differences between pregnant and lactating versus non-pregnant women, indicating that boosting could overcome the attenuated immune responses to the first dose during pregnancy, supporting the importance of vaccine boosting. Moreover, lactating women had a unique increase in natural killer (NK) cell-activating antibodies following the second dose of the vaccine. The authors observed some differences in responses to each mRNA vaccine formulation. Notably, mRNA-1237 induced immune responses that were enriched for neutrophil and NK cellrecruiting antibodies, whereas BNT162b2 slightly enriched for less specific IgG1 and Fc $\curlyvee$ RIIIa-binding antibodies, findings which were more prominent in pregnant and lactating women than in non-pregnant women. Further exploring these differences could inform future vaccine strategies to elicit antibody responses in pregnancy that would best protect the mother-infant dyad. Whether the coordination of immune responses between the two mRNA vaccine platforms results in different clinical outcomes remains to be determined, but using similar strategies as those demonstrated by this work would be helpful for rational maternal vaccine design.

With regards to passive antibody transfer, Atyeo et al. (5) observed a higher abundance of SARS-CoV-2 antibodies in maternal compared to cord sera, suggesting low transplacental antibody transfer rates to the fetal circulation. However, since all women in the study were immunized in the third trimester, this reduction in transfer is likely attributable to the timing of vaccination in later pregnancy, as noted by the authors. The authors propose that this reduction in transfer may result from a lower abundance of Fc $\gamma$ RIIIa binding antibodies in pregnant women, leading to decreased transfer efficiency across the placenta. Importantly, lactating women's breastmilk was highly enriched for antibodies capable of greater functional and FcR-binding qualities after vaccine boosting. These results imply that vaccination earlier in pregnancy and boosting later in pregnancy will help to maximize transplacental and breastmilk antibody transfer and show that immunization across different stages of gestation results in unique antibody profiles.
Atyeo et al. demonstrate how non-pregnant, pregnant, and lactating women diverge in their respective responses to mRNA-based immunizations against SARS-CoV-2, yet show that vaccine boosting remains an effective strategy to equalize the quality of antibody responses across groups. It is clear from this systems serology analysis that characterizing humoral responses following vaccination can greatly enhance our understanding of maternal immunity during pregnancy and inform vaccine administration strategies.

Fetal Sex and Maternal Immunity to SARS-CoV-2 Infection

In a complementary study, Bordt et al. (6) investigated the antibody and antiviral interferon responses in SARS-CoV-2 uninfected and infected pregnant women and whether fetal sex modulated these responses. Given that the placenta is a fetal-derived tissue, understanding how fetal sex might impact immune responses and transplacental antibody transfer could shed light on sex-associated differences in COVID-19 outcomes in infants and children.

Bordt et al. quantified anti-SARS-CoV-2 antibody titers, specificities, and functions, in the maternal and cord blood sera of pregnancies with male and female fetuses (Fig. 1B). The group found that mothers carrying male fetuses had lower maternal titers of IgG antibodies against all specific SARS-CoV-2 antigens tested. This finding of dampened immune responses to SARS-CoV-2 in mothers with male fetuses suggests that fetal sex modifies maternal antibody responses to infection during pregnancy. Additionally, the transfer ratio of SARS-CoV-2 antibodies into cord blood was lower in male versus female pregnancies. Importantly, the authors found that transplacental transfer of IgG against other pathogens, such as those causing pertussis and influenza, was not reduced in male versus female pregnancies.

The authors further explored whether fetal sex-specific differences in placental FcR expression modulated the observed decrease in SARS-CoV-2 IgG transfer. Placental staining and gene expression analyses revealed sexually dimorphic expression of placental FcRs with an up-regulation of FcRn, Fc $\gamma R I I$, and Fe $\gamma$ RIII, and an increase in co-localization of FcRn and Fc $\gamma$ RIII in male-derived placentas. Given that this increase in placental FcR expression and co-localization should theoretically enhance transplacental IgG transfer, the authors examined alternative hypotheses as to why transport of maternal SARS-CoV-2 IgG into cord blood was reduced in male pregnancies. Glycan profiling revealed that SARS-CoV2-infected mothers with male pregnancies produced higher titers of antibodies modified by fucosylation and galactosylation. As fucosylated antibodies are less efficiently transferred by Fc $\curlyvee$ RIII receptors, these findings help to elucidate why SARS-CoV-2 IgG transfer was decreased in male pregnancies (9).

Overall, the findings of this study suggest dynamic and 
divergent immune responses to SARS-CoV-2 in pregnant women based on fetal sex. Mothers with male fetuses had lower SARS-CoV-2 antibody titers with differential FcR binding and glycosylation following infection and lower rates of anti-SARS-CoV-2 IgG transfer across the placenta. These observations have important implications for SARS-CoV-2 vaccination in male- versus female-expectant mothers and, following parturition, the care of at-risk male children. It is essential that we continue to study these dichotomous fetal sex responses to infection in pregnancy across gestation.

\section{Implications for Translation}

Infections during pregnancy contribute to high rates of maternal and fetal morbidity and mortality. However, the study of maternal-fetal immune responses to such infections remains underwhelming. The studies by Atyeo et al. and Bordt et al. on the immune responses to SARS-CoV-2 vaccination and infection demonstrate the critical nature of investigating maternal-fetal immune responses during pregnancy, a highly unique biological state. The findings of these studies have direct clinical implications for the COVID-19 pandemic and future maternal-fetal vaccination strategies, as they reveal that pregnant and lactating women have distinct immune responses to immunization and natural infection. Furthermore, these studies echo the call-to-action to incorporate women at different stages of gestation into clinical trials, thereby increasing their representation in the development of vaccines.

\section{REFERENCES AND NOTES}

1. K. J. Gray, E. A. Bordt, C. Atyeo, E. Deriso, B. Akinwunmi, N. Young, A. M. Baez, L. L. Shook, D. Cvrk, K. James, R. De Guzman, S. Brigida, K. Diouf, I. Goldfarb, L. M. Bebell, L. M. Yonker, A. Fasano, S. A. Rabi, M. A. Elovitz, G. Alter, A. G. Edlow, Coronavirus disease 2019 vaccine response in pregnant and lactating women: A cohort study. Am. J. Obstet. Gynecol. 225, 303.e1-303.e17 (2021). doi:10.1016/i.ajog.2021.03.023 Medline
2. A. Schäfer, F. Muecksch, J. C. C. Lorenzi, S. R. Leist, M. Cipolla, S. Bournazos, F. Schmidt, R. M. Maison, A. Gazumyan, D. R. Martinez, R. S. Baric, D. F. Robbiani, T. Hatziioannou, J. V. Ravetch, P. D. Bieniasz, R. A. Bowen, M. C. Nussenzweig, T. P. Sheahan, Antibody potency, effector function, and combinations in protection and therapy for SARS-CoV-2 infection in vivo. J. Exp. Med. 218, e20201993 (2021). doi:10.1084/jem.20201993 Medline

3. D. K. Kaye, The moral imperative to approve pregnant women's participation in randomized clinical trials for pregnancy and newborn complications. Philos. Ethics Humanit. Med. 14, 11 (2019). doi:10.1186/s13010-019-0081-8 Medline

4. C. J. Megli, C. B. Coyne, Infections at the maternal-fetal interface: An overview of pathogenesis and defence. Nat. Rev. Microbiol. $\cdots, 1-16$ (2021). Medline

5. C. Atyeo, E. A. DeRiso, C. Davis, E. A. Bordt, R. M. DeGuzman, L. L. Shook, L. M. Yonker, A. Fasano, B. Akinwunmi, D. A. Lauffenburger, M. A. Elovitz, K. J. Gray, A. G. Edlow, G. Alter, COVID-19 mRNA vaccines drive differential Fc-functional profiles in pregnant, lactating, and non-pregnant women. Sci. Transl. Med. 13, eabi8631 (2021). Medline

6. E. A. Bordt, L. L. Shook, C. Atyeo, K. M. Pullen, R. M. De Guzman, M.-C. Meinsohn, M. Chauvin, S. Fischinger, L. J. Yockey, K. James, R. Lima, L. M. Yonker, A. Fasano, S. Brigida, L. M. Bebell, D. J. Roberts, D. Pépin, J. R. Huh, S. D. Bilbo, J. Z. Li, A. Kaimal, D. Schust, K. J. Gray, D. Lauffenburger, G. Alter, A. G. Edlow, Sexually dimorphic placental responses to maternal SARS-CoV-2 infection. Sci. Transl. Med. 13, eabi7428 (2021). Medline

7. G. Mor, P. Aldo, A. B. Alvero, The unique immunological and microbial aspects of pregnancy. Nat. Rev. Immunol. 17, 469-482 (2017). doi:10.1038/nri.2017.64 Medline

8. S. N. Langel, C. E. Otero, D. R. Martinez, S. R. Permar, Maternal gatekeepers: How maternal antibody Fc characteristics influence passive transfer and infant protection. PLOS Pathog. 16, e1008303 (2020). doi:10.1371/journal.ppat.1008303 Medline

9. T. Li, D. J. DiLillo, S. Bournazos, J. P. Giddens, J. V. Ravetch, L.-X. Wang, Modulating IgG effector function by Fc glycan engineering. Proc. Natl. Acad. Sci. U.S.A. 114, 3485-3490 (2017). doi:10.1073/pnas.1702173114 Medline

Acknowledgments: Funding: E.C.S. is supported by the Medearis CMV Scholars Program and C.B.C. is supported by NIH grants AI081759, Al150151, Al145828, and Al145296.

Published First Release 19 October 2021

10.1126/scitransImed.abm2070 
Fig. 1. Characterization of the maternal immune response to SARS-CoV-2 infection and vaccination. (A) Maternal antibodies are transferred across the placenta to the fetus by binding neonatal Fc receptors (FcRn) and $\mathrm{Fcr}$ receptors. The functional role of these antibodies include direct neutralization of antigens, antibodydependent complement deposition and activation, antigen opsonization, antibody-dependent cellular phagocytosis, and antibodydependent cell-mediated cytotoxicity. (B) Atyeo et al. (5) and Bordt et al. (6) applied a systems serology approach to characterize the humoral immune response to SARS-CoV-2 vaccination or natural infection in pregnancy. The authors used maternal and cord blood sera or breastmilk to measure anti-SARS-CoV-2 antibody titers, glycosylation patterns, FCR binding, and FCRmediated antibody functions. They also used placental biospecimens to characterize gene expression and cytokine release in the placenta of women infected with SARS-CoV-2. CREDIT: ASHLEY MASTIN/SCIENCE TRANSLATIONAL MEDICINE

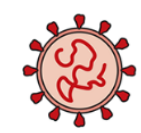

SARS-CoV-2 virus

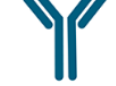

Antibodies
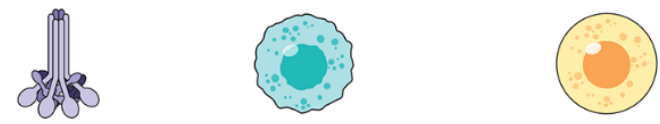

Complement Macrophage Natural killer (NK) cell

A

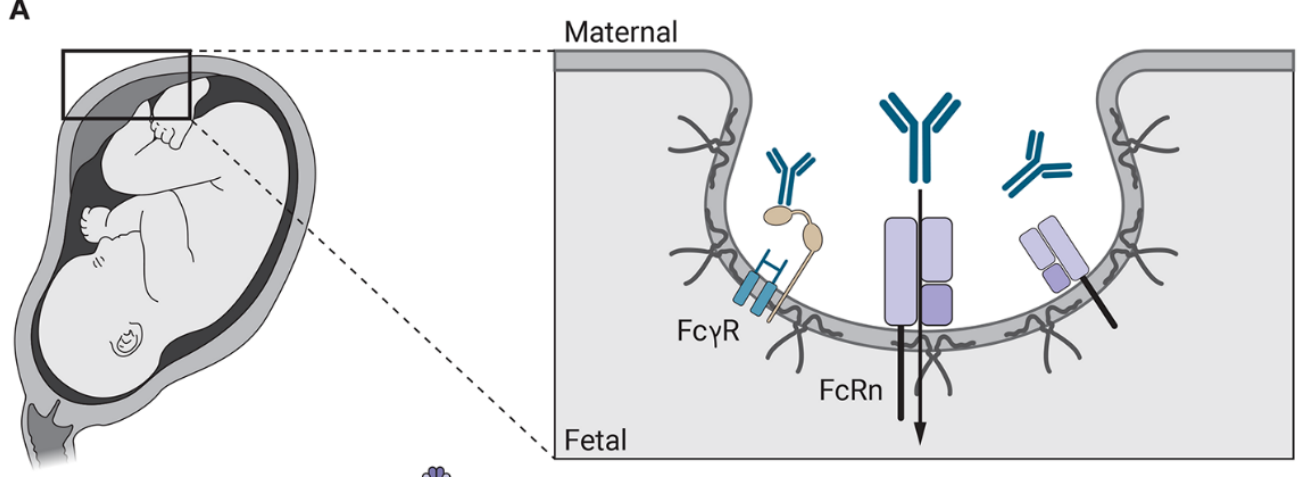

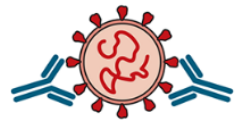

Neutralization

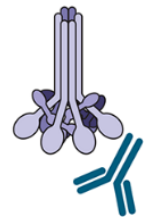

Antibody-dependent complement deposition and activation

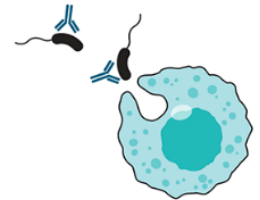

Opsonization and antibody-dependent cellular phagocytosis

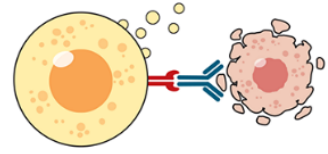

Antibody-dependent cell-mediated cytotoxicity
B

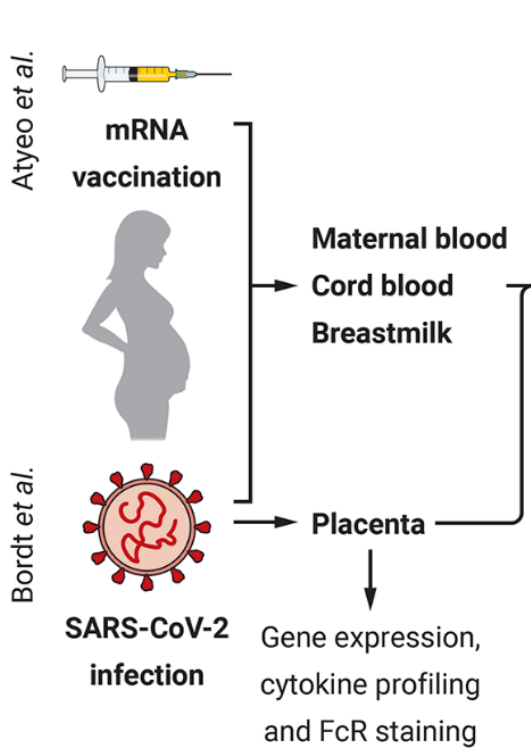

Systems serology approach

Incubate beads coupled to SARS-CoV-2 antigens with sera or breastmilk

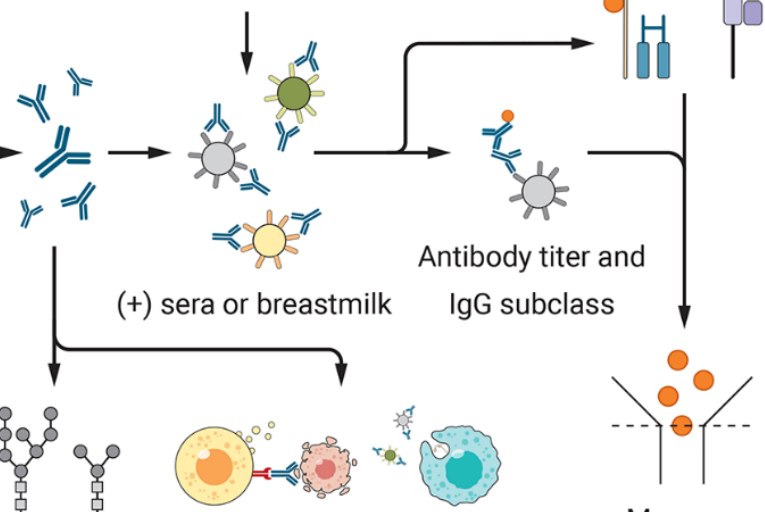

Fc glycan FcR-mediated profiling antibody functions
Measure

FcR binding Se

fluorescence

of beads 